Sociologie et sociétés

\title{
Les enfants de couples mixtes et leur rapport à leur pays étranger d'origine
}

\author{
Une identité « symbolique " affective fondée sur le lien de
} filiation

\author{
Nationally-mixed People and their Relationship to their \\ Foreign Country of Origin \\ An Affective "Symbolic" Identity Based on Family Ties
}

\section{Anne Unterreiner}

Volume 47, numéro 1, printemps 2015

URI : https://id.erudit.org/iderudit/1034426ar

DOI : https://doi.org/10.7202/1034426ar

Aller au sommaire du numéro

\section{Éditeur(s)}

Les Presses de l’Université de Montréal

ISSN

0038-030X (imprimé)

1492-1375 (numérique)

Découvrir la revue

\section{Citer cet article}

Unterreiner, A. (2015). Les enfants de couples mixtes et leur rapport à leur pays étranger d'origine : une identité « symbolique " affective fondée sur le lien de filiation. Sociologie et sociétés, 47(1), 249-273. https://doi.org/10.7202/1034426ar
Résumé de l'article

Étudier l'identité des enfants de couples mixtes permet d'éclairer les débats contemporains portant sur le contrôle de l'immigration et de l'intégration des populations issues de l'immigration. Une telle entreprise rend de plus visibles les facteurs susceptibles d'influencer l'identité nationale. Cet article s'attache à analyser le caractère " pratiqué » ou " symbolique » de l'identification nationale en référence au pays d'origine du parent migrant, à partir des discours identitaires d'une centaine d'enfants de couples mixtes rencontrés en France, en Allemagne et au Royaume-Uni. L'identification des enfants de couples mixtes à leurs origines nationales étrangères semble symbolique, c'est-à-dire élective plutôt que due à la pression communautaire. Elle se fonde sur l'affectif et le lien de filiation avant tout. Si au Royaume-Uni, cette identité symbolique s'ajoute à une multitude d'autres identités, en Allemagne, elle enrichit le sentiment d'appartenance au pays étranger d'origine existant. En France, elle compense l'absence d'identifications autres qu'en référence à la nation française.
Tous droits réservés @ Les Presses de l’Université de Montréal, 2015

Ce document est protégé par la loi sur le droit d'auteur. L'utilisation des services d'Érudit (y compris la reproduction) est assujettie à sa politique d'utilisation que vous pouvez consulter en ligne.

https://apropos.erudit.org/fr/usagers/politique-dutilisation/ 


\title{
Les enfants de couples mixtes et leur rapport à leur pays étranger d'origine
}

Une identité «symbolique» affective fondée sur le lien de filiation

\author{
A N N E U NTERREINER \\ Sciences Po, Observatoire sociologique du \\ changement (OSC), CNRS \\ 98, rue de l'Université, 75007 Paris, France \\ Courriel: anne.unterreiner@sciencespo.fr
}

\section{INTRODUCTION}

$\mathrm{D}$

ANS UN CONTEXTE OÙ LA MiXité CONJUgAle est au cœur du débat de contrôle de l'immigration et de l'intégration des populations issues de l'immigration, la question de l'identité des enfants issus de ces unions se pose. Pour les uns, ils s'identifient uniquement à leur pays de résidence. Pour les autres, ils sont enfermés dans des enclaves ethniques auxquelles ils se sentent appartenir. Grâce à la perspective sociologique, il est possible d'étudier l'identité des enfants de couples mixtes, de prendre de la distance et d'éclairer ces débats passionnés.

Les enfants de couples mixtes, c'est-à-dire les individus ayant des parents nés dans des pays différents, peuvent potentiellement s'identifier à de multiples «groupes de référence» nationaux (Merton, 1997 [1957]). Ils représenteraient l'individu «pluriel» par excellence, car pouvant jouir de multiples «principes de socialisation» (Lahire,

1. Cet article est issu des résultats de ma thèse de sociologie intitulée «Liens sociaux et construction identitaire des enfants de couples mixtes: Une étude comparée en France, en Allemagne et au RoyaumeUni» (EHESS, 2012). Je tiens ici à remercier Beate Collet pour ses conseils avisés, ainsi que le Comité de rédaction et les évaluateurs externes de la revue Sociologie et sociétés. Cet article a aussi été fortement enrichi des échanges que j'ai pu avoir avec Solenn Carof et Rébecca Ndour que je remercie chaleureusement. 
1998). Se focaliser sur les enfants de couples mixtes permet de rendre visible ce qui ne l'est pas: les facteurs susceptibles d'influencer l'identité nationale.

Les théories sociologiques passées relatives aux enfants de couples mixtes ne s'accordent pas quant à leur identité. Si, pour certains, ce positionnement social conduit à la «marginalité» et à la crise identitaire (Stonequist, 1965 [1937]), pour d'autres, il est libérateur et produit des identités transnationales positives. Les «crosscultural kids» notamment s'identifieraient ainsi à un autre espace que national (Pollock et Van Reken, 2009 [1999]). Tandis que pour Gordon (1964), suivant la Première École de Chicago, les enfants de couples mixtes seraient pleinement «assimilés» à leur société de résidence, et ne s'identifieraient en conséquence qu’à elle, cette identification étant incompatible avec toute identification au pays d'origine du parent migrant. Enfin, d'après une enquête au sein des populations blanches aux États-Unis, la mixité conjugale conduirait à une moindre volonté de transmission d'une identité ethnique à leur progéniture comparée aux parents en union «endogame» (Alba, 1990). La mixité conjugale «confine[rait] l'expression de l'identité ethnique à des formes symboliques», car les engagements ethniques demandent un investissement quotidien et peuvent être à la source de conflits qui seraient «incompatibles» avec la mixité conjugale (Alba, 1990: 206). Ainsi, dans le cas des individus ayant des origines multiples, l'identification nationale au pays d'origine du parent migrant ne prendrait qu'une forme «symbolique ${ }^{2} »$. Ces conclusions sont-elles généralisables aux enfants de couples mixtes résidant en Europe?

L'enquête empirique effectuée en France, en Allemagne et au Royaume-Uni permet de tester la véracité de ces représentations contradictoires de l'identité des enfants de couples mixtes. La France, l'Allemagne et le Royaume-Uni sont des pays d'immigrations anciennes. Le Royaume-Uni était d'abord un pays d'émigration vers le Nouveau Monde et les colonies britanniques, et devint un pays d'immigration, ce qui le poussa à ajuster sa politique migratoire (Layton-Henry, 1985). Tout comme la France, pays d'immigration de main-d'œuvre plus ancienne que le Royaume-Uni et l'Allemagne, le Royaume-Uni a dû gérer les flux migratoires vers la métropole en provenance de ses colonies puis de ses anciennes colonies. Alors que l'Allemagne, après la Seconde Guerre mondiale, a dû faire face au "retour» d'individus considérés comme ayant une ascendance allemande. À ceci s'ajoute le fait que ces trois pays furent en demande de main-d'œuvre après la guerre.

Si ces trois pays ont des caractéristiques comparables, leurs politiques migratoires ont cependant été différentes. Dans l'optique de synthétiser les travaux comparatifs antérieurs, Safi (2007: 27-28) a mis en évidence l'existence de trois modèles nationaux. La France suivrait un modèle « universaliste de la nation civique», visant à l'intégration d'individus pouvant facilement devenir nationaux, et non de communautés. Tandis que l'Allemagne et le Royaume-Uni se fonderaient sur des modèles pluralistes, le

2. Par pays d'origine, j'entends le pays de naissance du parent migrant ou le pays étranger auquel l'enfant de couple mixte le rattache dans son discours. 
Royaume-Uni connaîtrait un «multiculturalisme inclusif», combinant politiques de discrimination positive et obtention de la nationalité en vertu du jus soli. Le modèle allemand en revanche serait celui de l'«exclusionnisme différencié». Ce dernier renvoie à l'intégration professionnelle des migrants, perçus comme temporaires, et donc exclus de la communauté nationale parallèlement à des politiques visant à favoriser leur retour.

D'après les conclusions de l'enquête E.F.F.N.A.T.I.S³ (2001), ces modèles d'intégration nationaux affectent l'intégration des descendants d'immigrés, et notamment leur sentiment d'appartenance nationale. Les descendants d'immigrés s'identifieraient plus au pays d'origine de leurs parents en Allemagne, alors qu'ils auraient des formes d'identification multiples dans les deux autres pays étudiés. La question se pose alors de savoir si ce résultat est confirmé dans le cas des enfants de couples mixtes.

Actuellement, ces trois pays semblent connaître une certaine convergence en matière de flux et de politique migratoire. D’une part, la part des personnes nées à l'étranger résidant sur le territoire de chaque pays est comparable. En 2013, elles représentaient $12,7 \%$ de la population totale résidant en Allemagne, 11,5\% en France, $12,2 \%$ au Royaume-Uni ${ }^{4}$. D’autre part, ces pays mettent en avant une «politique d'intégration» plutôt que des «contrats de travail» (Schnapper, 1992). De plus, les politiques relatives à la nationalité ont pris un tournant similaire. Elles se fondent à la fois sur le droit du sol et du sang et s'appliquent sur un territoire national restreint. Parallèlement, dans les trois pays, l'obtention de la nationalité et d'un droit de séjour est soumise à une bonne connaissance de la langue du pays et de ses «valeurs». Enfin, en Allemagne comme au Royaume-Uni, le multiculturalisme a été remis en question officiellement. Ainsi, en France, en Allemagne et au Royaume-Uni, on assiste à une convergence des discours et des politiques visant l'intégration civique a minima, voire l'assimilation au groupe majoritaire, mais aussi le caractère défensif du sentiment d'appartenance nationale (Unterreiner, 2012).

Cela dit, ces trois pays présentent toujours des différences importantes quant à leur définition du «nous» national. Alors qu'en France l'idéologie relative au modèle républicain français est mise en avant parallèlement à un discours ethnicisant de l'identité nationale française, le passé allemand marque toujours la fierté nationale du sceau du tabou, en Allemagne de l'Ouest tout au moins. Et si toute identification «raciale» est difficile en Allemagne pour cette même raison, alors même qu'être Ausländer (étranger) est lié à la visibilité de son origine étrangère, au Royaume-Uni, elle est devenue une catégorie d'identification de l'État britannique, sous couvert d'«ethnicité». De plus, «race» et discours sur l'identité nationale sont intrinsèquement liés, que l'appartenance nationale exclue les non-Blancs, ou qu'elle les inclue dans un État multi - ethnique, ou — racial. Ainsi, que ce soit en France, en Allemagne et au Royaume-Uni, le contexte de vie des enfants de couples mixtes semble fortement

3. Acronyme de Effectiveness of National Integration Strategies towards Second Generation Migrant Youth in Comparative European Perspective.

4. Calculs effectués par l'auteur sur la base des données d'Eurostat. 
différer de la réalité observée dans les enquêtes menées à Montréal auprès de familles mixtes, au sein desquelles le multilinguisme et la multiculturalité sont fortement valorisés (Meintel, 2002).

Après avoir posé le cadre théorique et méthodologique de cette analyse, je mettrai en évidence les déterminants de l'identification nationale des enfants de couples mixtes à l'égard de leur pays étranger d'origine.

\section{APPROCHES THÉORIQUES ET MÉTHODOLOGIQUES}

Les enfants de couples mixtes, du fait de la pluralité de leurs groupes d'identification nationale potentiels, rendent visibles les dynamiques souterraines de l'identification nationale individuelle. Ils ont rarement été l'objet de recherches portant spécifiquement sur leur identification nationale ${ }^{5}$, et notamment leur identification à l'égard de leur pays étranger d'origine.

\section{Étudier l'identification au pays étranger d'origine...}

Nombre de recherches américaines et britanniques contemporaines portant sur l'identité des populations issues de l'immigration se concentrent sur l'identité «ethnique» ou «raciale» des «minorités ethniques» visibles (voir notamment Song, 2003). Contrairement aux populations blanches, ces populations devraient vivre dans un champ particulier de contraintes: les discriminations qu'elles subissent (Waters, 1990; Rumbaut et Portes, 2001). En réponse à ces discriminations, ces minorités ethniques développeraient une identité «réactive» (Rumbaut et Portes, 2001). Cette identification se fait en référence à un groupe défini socialement: la communauté ethnique résidant dans le pays de résidence, une minorité raciale telle que les Black Americans étudiés par Waters (1994), le pays de résidence ou encore le pays d'origine.

Étudier les enfants de couples mixtes définis selon leurs origines nationales permet d'apporter un regard nouveau sur la question de l'identité des personnes issues de l'immigration. Grâce à leur position sociale particulière, il est possible de voir comment l'individu s'identifie nationalement. L'objet de cet article n'est donc pas de savoir comment les enfants de couples mixtes réagissent en cas de discrimination, mais en vertu de quels registres ils s'identifient à leur pays étranger d'origine.

L'emploi du terme d'identité nationale est préféré à celui d'identité ethnique. Pour certains, ce dernier renvoie à l'identification raciale. Pour d'autres, à l'identification au pays d'origine de ses ancêtres. L'identification ethnique signifie également l'identification à une minorité ethnique dans le pays de résidence. L'identité ethnique renvoie donc à différents types d'identification, alors que le terme d'identité nationale se réfere uniquement à l'identification envers des groupes nationaux de référence. De même, parler d'identité culturelle me semble inadapté, car ce terme suppose l'existence d'une culture à laquelle l'individu s'identifie. Or, la question n'est pas ici de savoir quelle est

5. Les recherches de Lesbet et Varro (1995) et Khounani (2000) font figure d'exception. 
la culture de l'enfant de couple mixte, mais en vertu de quels registres il s'identifie à son pays étranger d'origine. Par identification nationale, il est entendu le rattachement subjectif de l'individu à un ou plusieurs groupes nationaux de référence. Si pour un individu lambda, celle-ci se superpose «naturellement» à la nationalité (au fait d'être le national d'un État) et à la citoyenneté (aux droits et devoirs politiques du citoyen d'un État), pour les enfants de couples mixtes, sentiment d'appartenance nationale, nationalité et citoyenneté peuvent renvoyer à des réalités différentes.

Gans a mis en avant qu'aux États-Unis, les troisième et quatrième générations de descendants de migrants européens peuvent développer une «ethnicité symbolique», sans qu'il soit pour autant question d'un «ethnic revival». Cette identité est définie comme étant une «allégeance nostalgique envers la culture de la génération migrante, ou celle du pays d'émigration; un amour et une fierté à l'égard d'une tradition qui peuvent être ressentis sans devoir être inclus dans le quotidien» (Gans, 1979: 9). Elle peut donc être mobilisée sans remettre en cause l'intégration au pays d'accueil.

[Elle] ne nécessite pas des groupes ou réseaux actifs; les sentiments d'identité peuvent être développés par allégeance envers des groupes symboliques qui ne se rencontrent jamais, ou à des collectifs qui se rencontrent seulement occasionnellement (...). De même, l'ethnicité symbolique ne nécessite pas une culture pratiquée, même si des symboles lui sont empruntés. (Gans, 1979: 12)

Ceci nous conduit à une première question de recherche: l'identification des enfants de couples mixtes à l'égard du pays étranger d'origine est-elle «pratiquée» ou «symbolique»?

Je définis l'identification nationale «pratiquée» avec le pays d'origine de son parent migrant comme étant l'identification à son «groupe d'appartenance» (Merton, 1997 [1957]). Dans ce cas, cette identité serait le fruit d'interactions et de pratiques quotidiennes, une identification «linéaire» pour reprendre les tenants de la théorie de l'assimilation segmentée (Portes et Rumbaut, 2001). S'identifier nationalement à son pays étranger d'origine s'imposerait en quelque sorte à l'individu. Cette identification pourrait être le fruit de la transmission intergénérationnelle ou de l'intégration dans la communauté ayant la même origine migratoire que l'enfant de couple mixte. La communauté correspond ici, suivant la définition de Barth (1969) de «groupe ethnique», au sentiment d'appartenance des enquêtés à un groupe existant dans le pays de résidence qui aurait une origine étrangère supposée commune.

A contrario, l'identification nationale symbolique à l'égard de son pays étranger d'origine est l'identification à un groupe national de référence qui ne correspond pas nécessairement au groupe d'appartenance de l'individu. Suivant la définition de la nation d'Anderson (2006 [1991]), cette identité est «imaginée» par l'individu. Au gré de ses envies, l'individu s'identifie à son pays étranger d'origine. Il «bricole», «s'invente des origines» pour reprendre l'expression de Ramos (2006). Cette «identité dormante», définie comme des «liens sentimentaux et symboliques» (Schnapper, 1991: 169), se fonde donc sur l'élection plutôt que sur la contrainte. Tout comme les secondes générations d’origine indienne rencontrées par Somerville (2008) au Canada, 
ou encore les Philippins de seconde génération étudiés par Wolf (2002), les enfants de couples mixtes pourraient choisir de s'identifier à leur pays étranger d'origine en vertu des liens affectifs qu'ils ont tissés.

Afin de répondre à la question du caractère pratiqué ou symbolique de l'identification au pays étranger d'origine, une enquête qualitative sur les enfants de couples mixtes a été effectuée.

\section{... à partir de l'analyse des discours identitaires des enfants de couples mixtes}

Les enfants de couples mixtes sont les descendants d'unions entre individus nés dans des pays différents, quel que soit le pays ${ }^{6}$, l'objectif étant d'étudier la manière dont les enfants de couples mixtes s'identifient à leur pays étranger d'origine.

Cette définition large permet de prendre de la distance par rapport aux définitions nationales de la mixité. Au Royaume-Uni par exemple, la mixité est conçue comme étant ethnique ou raciale, à quelques exceptions près ${ }^{7}$. Elle a été élaborée dans un rapport de domination intergroupe, blanc/migrant visible puis blanc/minorité ethnique, la socialisation interculturelle des «mixed race people» n'étant pensée que dans ce cadre. Bien que les recherches sur les «mixed race people» (voir par exemple Tizard et Phoenix, 1993; Ali, 2003) aient beaucoup apporté quant au processus d'identification raciale, aucune d'entre elles ne s'est concentrée exclusivement sur l'identité nationale. Cela dit, identités raciale et nationale peuvent toutes deux être présentes dans le discours de ces individus. Song (2010) a ainsi constaté la possible combinaison d'une identité culturelle britannique et d'une reconnaissance des origines. Et Aspinall et Song (2013) ont montré que différents facteurs déterminent l'«identité raciale et ethnique» des mixed race people: l'environnement local, la socialisation dans l'un ou l'autre pays, le regard d'autrui sur soi, et notamment les discriminations, l’origine sociale et la transmission intrafamiliale. Étant donné que les identités raciales et nationales sont souvent mêlées dans les discours identitaires individuels, il est difficile de conclure de ces recherches les déterminants de l'identification nationale de ces individus.

Déplacer le champ d'enquête vers les enfants ayant des parents nés dans des pays différents enrichit l'analyse de la mixité. Ne pas délimiter les «frontières » de la population étudiée par des pays sélectionnés a priori ouvre aussi d'autres perspectives d'analyses. Est-ce l'origine nationale ou plutôt le parcours migratoire du parent migrant qui le conduisent à transmettre tel ou tel « référent identitaire ${ }^{8} »$ ?

6. En France, en Allemagne et au Royaume-Uni, la nationalité à la naissance des parents telle que déclarée par les enfants de couples mixtes permet difficilement de rendre compte du type de socialisation parentale vécu.

7. Une exception notable est la recherche de Caballero et ses collègues (Caballero, Edwards et Puthussery, 2008).

8. Suivant la définition de l'identité proposée par Mucchielli selon qui elle serait «plurielle», «une affaire de significations données en fonction de leurs propres identités et de leurs engagements dans des projets, par l'acteur lui-même et/ou d'autres acteurs» (Mucchielli, 2009: 21), les «référents identitaires» 
Afin de répondre à ce questionnement, des enfants de couples mixtes âgés de 14 à 56 ans ont été rencontrés en France, en Allemagne et au Royaume-Uni entre 2008 et 2011. Comparer entre eux les discours d'adolescents et d'adultes permet de mettre au jour comment le parcours de vie influence le sentiment d'appartenance nationale (Unterreiner, 2015a). Ces entretiens semi-directifs ont été menés dans la langue nationale du pays dans trois pôles urbains multiculturels (la région de Francfort-sur-leMain, l'île-de-France et le Grand Londres) afin de comparer des contextes de résidence comparables. En effet, d'après différentes recherches ${ }^{9}$, vivre dans un environnement multiculturel permet le développement d'une identité positive. De plus, les recherches effectuées au Canada ont montré que vivre dans un tel environnement conduisait à des identifications multiples (Meintel, 1992).

C'est dans ces contextes nationaux particuliers que j'ai effectué 97 entretiens auprès d'enfants de couples mixtes; 41 personnes ont été rencontrées en France ${ }^{10}$, 27 au Royaume-Uni et 29 en Allemagne. Les entretiens ont été enregistrés, puis intégralement retranscrits, avant d'être analysés manuellement dans la langue de l'entretien, et traduits au moment de l'écriture.

\section{Tableau 1: Principales caractéristiques des enquêtés}

\begin{tabular}{|l|c|c|c|}
\hline & $\begin{array}{c}\text { Région } \\
\text { Île-de-France }\end{array}$ & $\begin{array}{c}\text { Région de Francfort- } \\
\text { sur-le-Main }\end{array}$ & $\begin{array}{c}\text { Région } \\
\text { du Grand Londres }\end{array}$ \\
\hline Nombre de femmes & 20 & 17 & 15 \\
\hline Âge moyen/Écart type & 36 ans/10,8 & 30 ans/10,6 & 32 ans/11,8 \\
\hline $\begin{array}{l}\text { Nombre d'enquêtés dont le parent migrant } \\
\text { est né en Europe' }\end{array}$ & 25 & 15 & 12 \\
\hline $\begin{array}{l}\text { Nombre d'enquêtés nés ou ayant vécu au moins } \\
\text { un an dans le pays d'origine de leur parent } \\
\text { migrant }\end{array}$ & 10 & 10 & 13 \\
\hline Nombre de binationaux (dont une trinationale) & 14 & 17 & 11 \\
\hline $\begin{array}{l}\text { Nombre d'enquêtés ayant au moins un parent } \\
\text { ayant un diplôme d'études supérieures }\end{array}$ & 20 & 14 & 23 \\
\hline $\begin{array}{l}\text { Nombre d'enquêtés ayant au moins un diplôme } \\
\text { de second cycle (Baccalauréat/Abitur/A-Level) ou } \\
\text { en cours de préparation de ce diplôme }\end{array}$ & 32 & 26 & 17 \\
\hline \begin{tabular}{l} 
Nombre d'actifs occupés \\
\hline
\end{tabular}
\end{tabular}

1. Quand les deux parents sont nés à l'étranger, les enfants de couples mixtes ayant au moins un parent né en Europe font partie de cette catégorie.

nationaux sont définis comme tous les éléments mobilisés par l'acteur pour s’identifier à une nation. Il peut par exemple s'agir de la langue, de la culture ou encore de la nationalité.

9. Voir par exemple Tizard et Phoenix (1993) et Saenz et al. (1995).

10. Dans le cas français, 30 des 41 entretiens mobilisés ici ont été effectués dans le cadre de la postenquête qualitative de l'enquête «Trajectoires et Origines» (INSEE — INED, 2008). 
Analyser des discours sur soi, des «discours identitaires», suppose de tenir compte du caractère proprement subjectif des déclarations des enquêtés quant à leur nationalité, leur niveau de langue ou encore le choix de leurs pairs. Ces trois éléments sont pris dans le discours identitaire de l'enquêté, plutôt qu'ils ne le déterminent. Après avoir demandé à l'enquêté de se présenter, une première question générale sur son rapport à la mixité lui a été posée. Des questions portant sur son parcours scolaire et professionnel ont suivi, avant que les thématiques de la langue, de la religion ainsi que de leurs transmissions soient abordées. Une fois la confiance de l'enquêté acquise, a suivi une question générale relative à la manière dont il se définit, puis sur celle dont il perçoit différents termes relatifs à l'identité, s'il est défini par autrui de la sorte, et s'il se définit en employant le terme mentionné. Il lui a ensuite été demandé de préciser comment il choisit ses amis et conjoint, avant de parler plus avant de ses relations intrafamiliales, de sa nationalité, et enfin, de l'existence de discriminations. Si tel est l'ordre des questions de ma grille d'entretien, une grande liberté était laissée aux enquêtés, qui ont pu mentionner ces points dans un autre ordre. Par cette méthode d'enquête, fondée sur l'analyse de discours et non l'observation, il est possible de voir quel est le rapport subjectif de l'individu à l'égard de son identité, plutôt que ses pratiques réelles.

L'analyse de ces discours identitaires révèle ainsi le caractère central de l'émotion dans le processus d'identification nationale au pays d'origine du parent migrant. Le «lien de filiation» (Paugam, 2008) se trouve au cour de ce processus dans les trois pays. C'est ainsi que la transmission a lieu d'une part, et qu'un lien affectif avec le pays d'origine du parent migrant se créé d'autre part. Afin de trancher la question du caractère symbolique ou pratiqué de cette identité nationale, nous verrons quelle est l'influence de «marqueurs identitaires» (Le Gall, 2003), la langue et la nationalité, et celle de la communauté sur l'identification nationale des enfants de couples mixtes à l'égard de leur pays étranger d'origine. Il nous sera ainsi possible de mettre au jour les différences nationales existant entre la France, l'Allemagne et le Royaume-Uni à cet égard.

\section{UNE IDENTITÉ AFFECTIVE FONDÉE SUR LE LIEN DE FILIATION}

Dans les trois pays, c'est par le lien de filiation et la résidence ou les vacances dans le pays étranger d'origine que les enfants de couples mixtes s'identifient à celui-ci.

\section{«Un lien sentimental avec une autre terre»}

S'identifier en signe de reconnaissance de ses ancêtres ou encore mettre en avant son «sang» dans le discours identitaire sont autant de signes de l'importance de l'émotion dans l'identification. Pour Leonor (24 ans $)^{11}$, dont le père est né au Pérou et la mère en France, être enfant de couple mixte signifie que «t'as un lien affectif, sentimental avec une autre terre en fait. Qui n'est pas celle où t’habites, mais... (réfléchit) mais...

11. Quand aucune précision n'est apportée, le pays de résidence est la France, l'Allemagne ou le Royaume-Uni, selon les origines nationales de l'enfant de couple mixte. Par exemple, si F. est Franco-Italien sans aucune précision quant à son lieu de résidence, il est supposé vivre en France. 
d'laquelle t'es issue quand même, d'une certaine façon. » Et Kenza (44 ans), une Franco-Algérienne, qui ne comprend pas l'arabe en dehors de quelques mots glanés çà et là, a fait le choix à 18 ans de demander la nationalité algérienne en signe de reconnaissance de son père, et d'une identité «moit-moit-». Le lien affectif qui l'unit à son père, plus que le lien politique ou la langue, est à la source d'une identification à l'égard de l'Algérie. L'émotion et le lien de filiation semblent donc être au cour de l'identification nationale à l'égard du pays étranger d'origine.

Parallèlement, des enfants de couples mixtes ont distingué entre une identité rationnelle et une identité émotionnelle, ressentie, physique. Marina (40 ans), qui a été élevée par sa mère allemande en Allemagne, se «sent» physiquement Indienne, mais est «conditionnée» comme une Allemande concernant sa manière de penser. Tandis que pour Christiane (33 ans), dont la mère est née en Italie et le père en Allemagne, le ressenti ne passe pas par le fait de mieux se sentir physiquement en Italie, mais par l'émotion ressentie lorsque des évènements, des odeurs ou autres lui rappellent son enfance où sa mère italienne était très présente. Les odeurs, comme celle du café, ou encore la langue italienne font que Christiane éprouve un sentiment de confiance, d'être chez elle. Tout cela la renvoie à sa mère, à son enfance. On pourrait ainsi dire qu'il s'agit de sa «madeleine», chère à Proust. Son «cœur», du fait de sa mère et de ce qu'elle a voulu transmettre penche vers l'Italie, mais ayant toujours vécu en Allemagne, son quotidien et sa Staatsangehörigkeit, sa citoyenneté, sont allemands.

Si l'identification au pays étranger d'origine est liée à l'émotion, elle est aussi fortement influencée par ce que les parents ont transmis à leurs enfants.

\section{La transmission parentale de référents identitaires}

Comme montré dans un précédent article (Unterreiner, 2014), les recherches antérieures portant sur la transmission au sein des familles mixtes se concentrent sur les déclarations parentales à ce sujet, voire leurs projets, plutôt que sur le discours des enfants issus de ces unions, sur ce qui leur a effectivement été transmis. Grâce à l'analyse des discours identitaires des enfants de couples mixtes, j'ai pu distinguer entre forte transmission parentale de référents identitaires propres au pays d'origine du parent migrant et absence de transmission.

La forte transmission est caractéristique des familles mixtes ayant des liens forts avec le pays d'origine du parent migrant du fait du parcours migratoire de celui-ci. Les enfants de couples mixtes dans ce cas ont, plus fréquemment que les autres, été socialisés dans les pays respectifs de leurs parents ou été scolarisés dans une institution leur permettant d'acquérir des connaissances du pays dans lequel ils ne résident pas. Quand les parents ont le projet de migrer dans le pays d'origine du parent migrant, ou le font effectivement, la transmission de référents identitaires relativement à ce pays est d'autant plus importante. Le père grec et la mère espagnole de Marieluz (34 ans) avaient le projet d'aller vivre en Grèce ${ }^{12}$. Marieluz a donc fréquenté, après l'école

12. Marieluz est née en Allemagne où elle a toujours vécu. 
maternelle allemande, une école primaire germano-grecque. Quand bien même elle se sent parfaitement «chez elle» en Allemagne, elle a été fortement imprégnée de référents identitaires grecs, plus qu'espagnols, ce qui pourrait notamment s'expliquer par le projet de migration en Grèce. De plus, en cas de va-et-vient effectifs du pays d'origine d'un parent à l'autre, les enfants de couples mixtes se sont vu transmettre des référents identitaires des deux pays.

Si elle ne se fait pas par l'institution scolaire ni par le va-et-vient dans les différents pays d'origine des parents, la transmission de référents identitaires de la part des parents migrants nécessite un fort engagement de leur part, mais aussi la capacité de le faire. C'est pourquoi ce sont les migrants adultes qui optent plus souvent pour cette démarche active de transmission. Ainsi, la variété de l'échantillon en termes de parcours migratoires parentaux met au jour l'importance de ce facteur dans la transmission à la génération suivante.

À l'opposé de cet axe de transmission se trouvent les enquêtés à qui aucun référent identitaire autre que ceux du pays de résidence n'a été transmis. Ce type est caractéristique des enfants de couples mixtes dont les parents migrants sont arrivés jeunes dans le pays où résident leurs enfants. Ayant eux-mêmes reçu des référents identitaires relativement à leur pays d'origine de manière partielle, ils ne se sentent pas à même de les transmettre. Arno (25 ans) est le fils de Klara qui s'est mariée à un homme né en Pologne ${ }^{13}$. Les parents d'Arno sont arrivés enfants en France. Il met en lumière que du fait de leur trajectoire migratoire et de l'absence de famille dans leurs pays de naissance et d'origine, ses parents lui ont donné une éducation à dominante française. L'éducation qu'Arno a reçue en France, la quasi-absence de transmission d'éléments culturels pouvant le rattacher à ses différents pays d'origine, et l'absence de famille pouvant faire le lien entre l'histoire familiale et son identité font qu'il estime ne pas pouvoir s'y identifier.

À ces migrants enfants s'ajoutent les migrants adultes pour qui la migration a marqué une rupture avec le passé. La cause de la migration ou leur forte volonté d'intégration à la société d'accueil a conduit à l'absence de transmission d'éléments de leur pays d'origine qui appartient au passé. À l'âge de 18 ans, la mère d'Angelina J. (23 ans) a migré du Zimbabwe au Royaume-Uni à la suite de la rencontre avec son père né au Royaume-Uni alors enseignant au Zimbabwe. Pour elle, cette migration a marqué un point de rupture avec ce pays, synonyme de souffrances. Parallèlement à cette vie difficile, le fait d'épouser un «homme blanc» n'a pas été accepté par sa famille. La mère d'Angelina J. n'a pas transmis le shona à sa fille pour éviter qu'Angelina J. ait le désir d'aller au Zimbabwe ${ }^{14}$. De plus, elle est fortement opposée au fait que sa fille voyage en Afrique, et à la démarche active vis-à-vis de ce continent qu'Angelina J. a entreprise.

13. Klara est née au Vietnam. Elle est le fruit de l'union d'un père légionnaire né en Hongrie, mais dont elle a appris récemment qu'il aurait une mère allemande, et d'une mère née en Chine et arrivée enfant au Vietnam. Elle est arrivée en France enfant, rejoignant sa mère qui s'était mise en couple avec un Français rencontré au Vietnam.

14. Le shona est l'une des langues officielles du Zimbabwe. 
L'âge ou les conditions de la migration des parents migrants peuvent donc conduire à une "intégration par assimilation» (Collet, 2003). Tel est le cas du père de Sophie F. (36 ans), née en France d'un père libanais et d'une mère française. Le père de Sophie F. a «embrassé la culture française (...) au détriment de sa culture libanaise» à son arrivée en France à 18 ans après avoir suivi une éducation française au Liban. Discriminé du fait de ses origines arabes, il avait pour projet identitaire pour sa fille que celle-ci devienne française et ne soit pas discriminée à son tour. Il ne lui a transmis aucun marqueur identitaire libanais, tel que la langue ou la nationalité libanaise. Du fait de cette éducation, de son pays de naissance et de socialisation, mais aussi de l'impossibilité d'aller au Liban en raison de la guerre et de la faiblesse de ses contacts avec sa famille libanaise, Sophie F. se définit comme française ${ }^{15}$. Ainsi les stratégies d'évitement des discriminations mises en place par les parents et l'importance des liens tissés par le parent migrant avant de migrer avec son pays d'accueil ont une importance capitale ${ }^{16}$.

L'absence totale du parent migrant dans l'éducation de l'enfant de couple mixte empêche toute transmission de référents identitaires relativement à son pays d'origine. Marina par exemple n'a pas connu son père indien dans son enfance. Rencontré brièvement lors d'un de ses passages à l'aéroport de Francfort-sur-le-Main lorsqu'elle avait 17 ans, elle l'a revu trois fois depuis. De ce fait, ce n'est pas par le canal de la transmission paternelle que son lien avec l'Inde a pu être tissé.

L'identité des enfants de couples mixtes en référence au pays d'identification de leur parent migrant est ainsi fortement liée au lien de filiation, par la transmission de référents identitaires mobilisables pour les plus jeunes et par le caractère affectif de ce lien. C'est pourquoi l'adéquation entre pays de résidence de la famille élargie et pays de naissance du parent migrant est centrale concernant l'identité nationale des enfants de couples mixtes.

\section{La qualité de la relation familiale dans le pays étranger d'origine}

Si du point de vue parental, la transmission de référents identitaires relativement au pays d'origine du parent migrant peut viser à tisser des liens forts avec la famille élargie (Le Gall et Meintel, 2011), du point de vue des enfants, ces mêmes liens permettent de s'identifier nationalement au pays étranger d'origine. En l'absence de transmission et de migration individuelle, la dimension affective de l'identité nationale passe en effet par le lien de filiation. Elle est déterminée par la qualité des relations familiales combinée avec le lieu de résidence de la famille élargie. On pourrait ainsi parler d'une identification symbolique au pays étranger d'origine pour les individus dont la force du lien de filiation a permis d'éviter une rupture totale avec ce pays.

15. Ceci dit, sa trajectoire migratoire personnelle a fait évoluer son sentiment d'appartenance nationale vers une identité multiculturelle, internationale.

16. Ceci confirme les résultats de recherches antérieures sur le sujet (Puzenat, 2008; Benson, 1981). 
Les enfants de couples mixtes rencontrés ayant développé une identité nationale affective forte avec leur pays étranger d'origine sont ceux dont les liens familiaux sont intenses et dont la famille élargie réside dans le pays d'origine du parent migrant. Tel est le cas de Chanel (25 ans) à l'égard de Trinidad ${ }^{17}$. Si Chanel se définit comme une «Black British Afro-Caribbean woman», et donc comme à la fois trinidadienne et nigériane, son identité nationale affective est orientée vers Trinidad. La force du lien de filiation maternel opposée à la faiblesse des liens au sein de la famille paternelle, à laquelle s'ajoute le fait que de nombreux membres de la famille paternelle ne résident plus au Nigéria, font que Chanel a développé une identité nationale affective forte à l'égard de Trinidad, tout en émettant le désir de mieux connaître le Nigéria. Chanel connaît beaucoup mieux Trinidad que le Nigéria, ayant été plus fréquemment dans le premier pays que dans le second. De plus, si l'idée de vivre dans le futur à Trinidad est envisageable pour Chanel, tel n'est pas le cas concernant le Nigéria. Ainsi, quand la transmission d'une identité nationale ne se fait pas par les parents, et quand les liens familiaux dans la famille du parent migrant sont faibles, les enfants de couples mixtes ne développent pas d'identité nationale affective à l'égard de ce pays d'origine.

L'identification nationale au pays étranger d'origine se fonde donc sur le lien de filiation et sur l'émotion qui lui est liée, ce qui tendrait à conclure qu'elle est symbolique.

\section{UNE IDENTITÉ PLUS SYMBOLIQUE QUE PRATIQUÉE}

Les enfants de couples mixtes pourraient aussi s'identifier au pays d'origine de leur parent migrant du fait de leur usage quotidien de référents identitaires, tels que la langue et la nationalité. Un autre indicateur du caractère pratiqué de leur identité serait le fait qu'ils orientent leurs choix amicaux et conjugaux vers des personnes de même origine étrangère qu'eux. Enfin, on pourrait conclure qu'ils pratiquent leur identification à leur pays étranger d'origine s'ils s'investissent fortement dans le groupe d'origine du parent migrant.

\section{Choisir de faire référence à la langue ou à la nationalité pour s'identifier}

D’après les adolescents rencontrés par Varro et Lesbet (1995: 169), «sans bilinguisme, point de biculturalité». Le discours des enquêtés rencontrés ici tend cependant à nuancer l'hypothèse d'un strict lien entre pratiques linguistiques et identification nationale.

La langue renforce certes le lien entre l'enfant de couple mixte et son pays étranger d'origine. Par la langue, il est possible de communiquer avec les membres de sa famille, consolidant de ce fait les liens intrafamiliaux. De plus, la langue est un canal de transmission de normes et valeurs. Xavier (20 ans), dont la mère est née en Colombie et le père au Vietnam, est né en France où il réside toujours. Son apprentissage progressif

17. Chanel réside au Royaume-Uni où elle est née. 
de la langue espagnole grâce à sa mère et à l'école lui a permis de tisser des liens plus forts avec des membres de sa famille maternelle, de se rapprocher de la culture colombienne et donc de s'y identifier plus fortement qu'auparavant. Xavier se sent colombien du fait de sa double nationalité obtenue il y a deux ou trois ans, d'être allé en Colombie à 8,15 et 18 ans, et d'avoir pu se rapprocher du pays et de sa famille là-bas grâce à ses progrès en espagnol.

A contrario, la non-maîtrise de la langue d'origine du parent migrant est un argument mis en avant pour ne pas s'identifier à son pays d'origine. André A. (48 ans) ne parle pas polonais. Sa mère a été scolarisée au lycée français en Pologne, et se rendait fréquemment en France en vacances. Le père de celle-ci y possédait de nombreuses propriétés. La mère d'André A. a aussi étudié un an dans un lycée en France avant sa migration. La Seconde Guerre mondiale a éclaté alors qu'elle était en vacances en France avec son frère et sa mère. Les liens avec la Pologne ont alors été rompus. La mère d'André A. se considère comme française avant tout et n'a de ce fait pas transmis la langue polonaise à ses enfants. Pour André A., la Pologne correspond donc au passé, au fait de savoir qu'il a des «ORIGINES polonaises ${ }^{18}$ », mais pas au présent. Ce «lien historique» qu'il a avec la Pologne ne lui permet pas de s'y identifier.

La langue est un référent identitaire symbolique qui peut être mobilisé dans le discours identitaire des enquêtés quel que soit leur niveau de langue, sans pour autant que l'on puisse considérer la langue comme une condition préalable nécessaire et suffisante à l'identification nationale. Premièrement, le bilinguisme n'est pas toujours mobilisé dans le discours identitaire. Karin (52 ans), née en France où elle réside toujours d'un père français et d'une mère allemande, a fait le choix de ne pas s'identifier à l'Allemagne au début de l'âge adulte, malgré son bilinguisme. Deuxièmement, l'absence de bilinguisme n'est pas toujours synonyme d'absence d'identification. En effet, Aicha (15 ans) dont le père est marocain et la mère britannique ne parle pas arabe pour l'instant. Pourtant, elle se définit comme half and half et marocaine. Troisièmement, si la langue peut être mobilisée pour s’identifier à son pays étranger d'origine, la volonté de s'identifier à ce pays peut aussi conduire les enfants de couples mixtes à apprendre une langue. Theodora (40 ans), qui réside en Allemagne, dont le père est né en Allemagne et la mère en Grèce, a ainsi commencé à apprendre le grec sur le tard. Enfin, la langue apprise dans le but de créer un lien avec la terre étrangère de ses ancêtres peut ne pas être la langue maternelle du parent migrant. Marina suivait par exemple des cours d'hindi au moment de notre rencontre alors que ce n'est pas la langue d'origine de son père né en Inde.

Ainsi, la connaissance de la langue du parent migrant permet de renforcer les relations intrafamiliales et d'acquérir une connaissance des normes et valeurs de ce pays, facilitant l'identification à ce dernier. Mais il n'est pas possible pour autant d'établir un lien direct entre bilinguisme et identification nationale, et absence de bilin-

18. Lorsque des mots sont en lettres capitales dans les extraits d'entretien, cela signifie que le locuteur met l'accent sur ceux-ci. 
guisme et d'identification. Si la correspondance entre niveau de langue et identification nationale au pays étranger d'origine n'est pas stricte, c'est aussi parce d'autres référents identitaires peuvent être mobilisés dans le discours identitaire, tels que la nationalité.

Or, tout comme la langue, la nationalité semble avoir une valeur symbolique plutôt que de se superposer strictement au sentiment d'appartenance nationale des enfants de couples mixtes. La double nationalité est en effet évaluée par les enfants de couples mixtes selon deux prismes quelle que soit leur nationalité effective: un signe ou une volonté de reconnaissance de ses origines ou de son identité pour les uns, ou selon son degré d'utilité pour les autres ${ }^{19}$.

Ces derniers ont une vision pragmatique de la nationalité, des droits qu'elle pourrait conférer ou non. Teo (39 ans) par exemple tire les bénéfices de sa double nationalité: grâce à elle, il lui est possible de vivre en dehors du Venezuela ${ }^{20}$, pays dans lequel il a été fortement discriminé du fait de son apparence physique et de son comportement atypique. S’il devait choisir entre ses nationalités allemande et vénézuélienne, il opterait pour la première, sans pour autant se sentir allemand, mais parce que sans elle, il serait condamné à vivre au Venezuela. Dans cette perspective, le souhait d'obtenir la nationalité du pays du parent migrant est justifié par les droits que cela ouvre (droit de séjour, droit de succession, droit de propriété par exemple).

Mais tous les enfants de couples mixtes n'ont pas cette vision pragmatique de la nationalité. D'autres y attachent une importance symbolique, et l'utilisent comme référent identitaire en signe de reconnaissance de leurs origines, de leurs parents ou de leur identité renvoyant à leurs deux pays d'origine ${ }^{21}$. La volonté de demander, ou la demande effective, de nationalité peut aussi être motivée par la reconnaissance symbolique d'une identité ou d'une origine qu'une telle nationalité apporterait. Angelina J. aimerait pouvoir demander la nationalité zimbabwéenne, en signe de reconnaissance de sa double appartenance nationale: «[C] 'est juste l'idée d'avoir ce dual heritage, cette double nationalité que tu ressens que tu fais vraiment partie de l'autre pays aussi au niveau du passeport. (...) Je ressens, tu vois, c'est là que j'appartiens, c'est deux endroits, pas que mon passeport anglais.»

Le rapport à la nationalité du parent migrant semble d'autant plus symbolique qu'elle n'est pas vécue comme une contrainte liée aux devoirs de citoyens envers le pays du parent migrant. Concernant le fait de jouir de la nationalité du parent migrant, les droits qui y sont liés sont plus fréquemment mis en avant que les devoirs: droit de séjour, liberté de voyage à l'étranger accrue, droit de propriété, droit de succession, ou encore droit de vote. Ce dernier est rarement utilisé, car vécu comme illégitime. Il a

19. Dans la présente analyse, les deux types d'argumentaires sont scindés, mais ils peuvent se combiner dans celui d'un même enquêté.

20. La mère de Teo est originaire du Costa Rica et du Nicaragua et son père d'Allemagne. Teo est né au Venezuela où il a vécu jusqu'à l'âge de 20 ans. Il a ensuite résidé deux ans en Allemagne, avant de retourner vivre au Venezuela pour deux ans. Il est alors parti vivre à Londres, où il habite depuis, à l'exception d'une tentative de retour au Venezuela pour deux ans et demi avec sa femme vénézuélienne et leurs enfants.

21. Cette identité est qualifiée d'«identité d'héritier» (Unterreiner, 2015a). 
semblé contestable pour Amber ( 35 ans) de participer à la prise de décision politique sans être pleinement informée des tenants et aboutissants ni en subir les conséquences. Amber a vécu à la fois aux États-Unis et au Royaume-Uni, où elle réside au moment de l'entretien. Elle choisirait la nationalité britannique plutôt qu'américaine si elle devait renoncer à l'une d'elles car elle estime la première plus «sûre», habite au Royaume-Uni et ne se sent pas citoyenne américaine.

Ceux qui se sentent citoyens de leur pays étranger d'origine, c'est-à-dire comme ayant aussi des devoirs, sont parmi ceux qui y ont vécu suffisamment longtemps pour estimer que ces devoirs sont légitimes. C'est ainsi que Samantha met en avant qu'en tant que binationale, elle est citoyenne à la fois des États-Unis et du Royaume-Uni. Samantha (25 ans) a vécu aux États-Unis, pays auquel elle s'identifie, jusqu'à ce qu'elle vienne poursuivre ses études au Royaume-Uni, où elle vit depuis quatre ans. Voter, payer ses impôts, être jurée dans les deux pays, être attentive «à ce qui s'passe dans les deux pays», mais aussi faire du bénévolat et pouvoir travailler au Royaume-Uni font d'elle une citoyenne américaine et britannique. De même Dervish (34 ans) 22 , qui a vécu à la fois en Allemagne et en Turquie, serait prêt à se battre pour défendre ses deux patries en temps de guerre.

Prendre part à la vie de la Cité et assumer la charge incombant au citoyen semble être profondément influencé par la résidence, plutôt que par le lien de filiation par lequel la nationalité serait transmise. Ceci explique aussi pourquoi certains enfants de couples mixtes ne concluent pas de leur nationalité qu'ils sont citoyens de leur État étranger d'origine et mettent en place des stratégies d'évitement (renoncer à sa citoyenneté, ou encore ne pas la demander) à l'égard des devoirs qui leur incombent. À 18 ans, Stefan (39 ans), qui réside en Allemagne sous le même toit que sa mère allemande, a voulu se rendre en Irak avec sa sœur pour se recueillir sur la tombe de son père né dans ce pays, enterré dans le tombeau familial. Ils se sont alors informés auprès des autorités de la faisabilité de ce voyage ${ }^{23}$, Saddam Hussein étant au pouvoir à l'époque. Stefan a alors appris qu'il avait la nationalité irakienne, qu'il risquait d'être enrôlé dans l'armée irakienne et d'être contraint de rester en Irak, d'où son choix de ne pas y aller. Être national du pays de son parent migrant ne signifie donc pas que les enfants de couples mixtes participent ou souhaitent participer activement à la vie politique de cette nation.

Ainsi, être citoyen de son pays étranger d'origine peut être vécu sur le mode utilitariste: jouir de droits et libertés supplémentaires, être protégé par l'État, sans pour autant vouloir remplir ses devoirs de citoyen, d'une part. D'autre part, cette nationalité peut renvoyer à une logique de reconnaissance symbolique de ses origines, voire d'une

22. Dervish est né en Turquie. Il est arrivé bébé avec sa mère allemande en Allemagne, après la séparation du couple parental. Vers l'âge de 10 ans, il est retourné vivre chez son père en Turquie, du fait des difficultés financières de sa mère. À 23 ans, il est parti vivre aux États-Unis mais la crise économique et l'annulation de son visa l'ont conduit à quitter le pays trois ans plus tard. Les perspectives d'emploi étant plus nombreuses en Allemagne qu'en Turquie, il est alors venu y travailler.

23. Stefan a un doute sur le fait que ce soit les autorités irakiennes ou allemandes. 
identité d'héritier (Unterreiner, 2015a). C'est parce que la nationalité n'est pas vécue comme une contrainte que tous les enfants de couples mixtes ne lui accordent pas la même valeur.

La langue et la nationalité influencent l'identification nationale au pays étranger d'origine. Mais l'inverse est vrai aussi. C'est pourquoi on peut qualifier ce sentiment d'appartenance nationale de symbolique. Il semble en effet être plus fondé sur l'élection que sur les pratiques quotidiennes. Le caractère symbolique plutôt que pratiqué de l'identité nationale relativement au pays étranger d'origine est aussi perceptible au regard des choix de pairs.

\section{L'indifférence à l'égard de l'origine nationale des pairs}

Choisir ses amis et partenaires selon leur origine et être contraints par eux d'avoir un certain comportement pourraient influencer l'identification nationale des enfants de couples mixtes. Or, il apparaît de leurs discours que la majorité des enfants de couples mixtes évoluent dans un environnement leur permettant d'effectuer des choix amicaux et conjugaux sans tenir compte de l'origine nationale. Ceci renforce le caractère symbolique plutôt que pratiqué de leur identification à leur pays étranger d'origine.

Les affinités, la personnalité sont les critères premiers mis en avant concernant le choix des pairs. Le point de vue de Manu (36 ans), dont la mère est née en France et le père au Vietnam, est typique de cette posture. À la question sur les origines de ses amis, il répond: «franchement y a pas, y a pas un truc qui ressort en particulier. (...) C'est pas parce qu'ils sont de... (inspiration) d'Afrique..., ou de... d'Asie ou d'machin, que voilà, tout d'suite ça va être mon pote. Non. (acquiescement) On est POTES, PARCE QUE on se retrouve quelque part euh... des points communs, euh... à partager, qu'ça soit d'la musique, je sais pas des sorties, 'fin des goûts communs, et c'est pour ça qu'ce sont mes amis.»

C'est l'environnement de résidence qui importe plutôt que l'orientation vers une origine nationale ou une autre. Quand les amis ou les conjoints ont été rencontrés dans le pays de résidence, les enfants de couples mixtes déclarent à de rares exceptions près que ceux-ci sont majoritairement originaires soit du pays de résidence, soit qu'aucune origine ne prévaut. L'origine du parent migrant et le fait d'avoir des liens familiaux dans son pays d'origine ne permettent pas à eux seuls de choisir des amis de même origine que celle du parent migrant. L'origine nationale des camarades de classe, puis des collègues de travail, et des voisins, bref de l'environnement dans lequel l'enfant de couple mixte se socialise est déterminant. L'origine des amis de Sophie J. (34 ans), dont la mère est née en Guyane britannique et le père en Écosse, correspond à celle des milieux dans lesquels elle a évolué. Si à l'école primaire, Sophie J. se sentait très différente et était isolée, elle a tissé des liens d'amitié à l'école secondaire qu'elle qualifie de «beaucoup plus mixte $[s]$ ». Puis a suivi l'université qui était «à nouveau (...) très blanc [he]».

Les personnes ayant déclaré avoir des amis dans leur pays étranger d'origine comptent parmi celles qui y ont vécu ou celles qui y séjournent en vacances suffisam- 
ment longtemps et fréquemment pour y tisser des liens en dehors de la cellule familiale. Jonny (19 ans), dont la mère est née en Allemagne et le père en Tunisie, part en vacances une ou deux fois par an en Tunisie. Il est ami avec cinq-six Tunisiens de «[son] quartier». Toutefois, même s’il maintient des liens avec eux grâce à internet quand il n'y est pas, il est moins proche d'eux que de ses amis résidant en Allemagne qui eux sont principalement «Ausländer», mais aussi Allemands.

Les enfants de couples mixtes déclarent majoritairement leur indifférence à l'égard de l'origine de leurs amis et conjoint. Viennent ensuite ceux qui affirment que les personnes d'origines étrangères, que celles-ci soient mixtes ou non, sont source d'enrichissement ou permettent de se constituer un entre-soi protecteur. Peu d'enfants de couples mixtes accordent de la valeur à la mixité en tant que telle. Mais si un sentiment de proximité à l'égard d'autres enfants de couples mixtes peut être ressenti, il ne conduit pas nécessairement à orienter leurs choix conjugaux ou amicaux vers d'autres enfants de couples mixtes. Les discriminations subies peuvent aussi faire que l'enfant de couple mixte ressente une proximité avec des individus ayant une origine migratoire. Silad (19 ans), dont la mère est née en Indonésie et le père en Allemagne, estime que $90 \%$ de ses amis ont une origine étrangère, ce qui lui permet de partager ses expériences de discrimination.

Mais si entre-soi protecteur il y a, on ne peut parler d'entre-soi communautaire, car celui-ci est multiculturel. Une minorité seulement d'enfants de couples mixtes cherchent à tisser des liens avec des individus ayant la même origine étrangère qu'eux, et ce, dans le cas où l'identification avec le pays d'origine du parent migrant est forte.

Si dans le cadre amical, les enquêtés se déclarent majoritairement aveugles à l'égard des origines, ou curieux à l'égard de l'autre, le choix du conjoint renvoie plus fortement aux frontières intergroupes. Une minorité d'enfants de couples mixtes orientent en effet explicitement leurs choix conjugaux vers un individu originaire de leur pays de résidence. Ce choix peut être effectué dans l'optique de suivre l'opinion parentale, comme l'explique Nicholas (31 ans). Fils d'une femme née au RoyaumeUni et d'un homme d'origine arménienne né en Irak, Nicolas dit ne pas chercher «consciemment» ses partenaires selon leur origine. Mais la volonté de ses parents pèse sur ses choix, cherchant plutôt « une partenaire (...) blanche et née anglaise, ou européenne».

D'autres enfants de couples mixtes évitent consciemment des partenaires ayant la même origine étrangère qu'eux. Tel est le cas de Djin ( 25 ans) dont la mère est née en Corée et le père en France par exemple. Il ne se projette absolument pas avec une femme coréenne, sa mère étant très critique à l'égard de ses congénères, et ayant observé leur comportement dans l'association de percussions coréennes dont il est membre.

Mais quels que soient les choix conjugaux ou amicaux pour lesquels les enfants de couples mixtes optent, ils ne semblent pas être guidés par la pression communautaire extrafamiliale. Et du fait de leurs choix de pairs, ils s'identifient majoritairement de manière symbolique à leur pays étranger d'origine, car celui-ci est peu présent dans leurs interactions quotidiennes. Ainsi, l'investissement dans la communauté de même 
origine que celle du parent migrant semble être, à l'âge adulte, le fruit d'un choix individuel plutôt que de la pression communautaire ou intrafamiliale.

\section{Le faible investissement auprès du groupe d'origine du parent migrant}

Les activités des enfants de couples mixtes dans des associations, des Églises, ou encore des écoles communautaires semblent être peu contraignantes. Elles pouvaient certes correspondre aux choix éducatifs parentaux pendant l'enfance. Mais celles pratiquées par les enquêtés au moment de l'enquête étaient le fruit de choix individuels et non pas d'une pression communautaire ou parentale.

Enfants, des enquêtés se sont vu imposer des activités dans la communauté migrante par leur parent migrant. Jusqu'au début de l'adolescence, Djin se rendait le dimanche au temple où le culte était en langue coréenne, suivi d'un repas et d'ateliers de jeunes dans l'après-midi. Parallèlement, la famille se rendait à l'église le samedi soir, son père étant catholique. Cette «double éducation» religieuse était vécue comme quelque chose qui était «plus embêtant qu'autre chose» pour Djin et son frère. Mais l'élection a progressivement pris le pas sur la contrainte. Djin vivait l'apprentissage de la langue coréenne comme une obligation imposée par sa mère jusqu'au collège. Tandis qu'à présent, il cherche à apprendre cette langue et à en savoir plus sur la culture coréenne, ce qui l'a conduit à devenir membre d'un groupe de percussions coréennes.

Les enfants de couples mixtes qui avaient des activités régulières dans des organisations communautaires au moment de l'enquête sont rares. Les activités sont plutôt de l'ordre du loisir, comme le fait de pratiquer la danse portugaise et de faire des «sorties» avec ses amis d'origine portugaise plus «typiques» pour Sylvia (25 ans), qui habite en France depuis toujours et dont le père est né au Portugal.

Ainsi, l'investissement communautaire semble peu fréquent parmi les enfants de couples mixtes, et quand c'est le cas, il est occasionnel, non exclusif et peu contraignant. C'est ce que confirment les cas de fort investissement communautaire.

Marieluz se présente comme étant d'abord «moi», et répond aux questions portant sur ses origines de la manière suivante: «Je suis halb Griechin, halb Spanierin, mais je suis née et ai GRANDI en Allemagne.» Marieluz a des contacts fréquents avec la Grèce pour raisons professionnelles. Elle a en effet repris le poste de son père dans l'entreprise familiale à la suite de son décès. Marieluz parle mieux grec qu'espagnol, ayant été scolarisée en école primaire germano-grecque, et le grec étant la langue principalement parlée à la maison. Si Marieluz n'a pas cherché ses amis à l'école ou à l'université en fonction de leur origine, elle a pratiqué pendant de nombreuses années la danse dans une association espagnole où ses amies étaient toutes espagnoles. Puis elle a développé un goût pour la musique grecque et a eu de nombreux amis grecs avec qui elle se rendait à des évènements grecs. Elle est de plus partie plus fréquemment en vacances en Grèce qu'en Espagne. Marieluz pense qu'il aurait été difficile pour elle de faire sa vie avec quelqu'un de «culture» différente de la sienne et se sent mieux avec des Grecs. Elle estime que le fait que la Grèce soit plus présente dans sa vie, sur le plan 
de la langue, de la religion et de ses activités professionnelles, a pu l'influencer dans son choix matrimonial, c'est-à-dire le fait d'épouser un Grec. Cela dit, elle a des amis de toutes origines.

Kevin (16 ans) est le fils d'une femme chinoise de Malaisie et d'un père né au Royaume-Uni dont les parents étaient de Hong Kong. Il vit dans un quartier de Londres proche d'une zone où la communauté chinoise est fortement implantée. Kevin se définit «ethniquement» comme chinois, est bénévole au sein de la «communauté chinoise», et se sent plus proche de ses pairs chinois, car ayant les mêmes «traditions» et la même «couleur de peau ». Toutefois, il éprouve des difficultés à s'identifier en tant que Chinois, car il ne parle pas bien ni le mandarin ni le cantonais, ce qui fait qu'il est regardé de haut par ses pairs chinois. De plus, Kevin a reçu une éducation «occidentale» de son père et «orientale» de sa mère, mais dit avoir été plus influencé par sa mère que par son père. Il se définit comme «British-Chinese», un «mix» à dominante chinoise. Le fait d'être entre-deux lui donne une certaine «liberté» à l'égard de la communauté chinoise, mais le place aussi à la marge de celle-ci, quand bien même elle fait partie de son quotidien.

Ces deux cas de forte insertion communautaire nous enseignent que d'une part, les enfants de couples mixtes n'ont pas des pairs uniquement de même origine que leur parent migrant, et d'autre part, qu'un sentiment d'extranéité à l'égard du groupe de même origine étrangère peut malgré tout voir le jour du fait de ne pas parler la langue de ces derniers.

\section{DISCUSSION : LES DISTINCTIONS NATIONALES EN MATIÈRE D'IDENTIFICATION SYMBOLIQUE}

L'analyse des discours identitaires des enfants de couples mixtes a montré que l'identification nationale à leur pays étranger d'origine est plus de l'ordre du symbolique que de la pratique. Cette identité symbolique se développe grâce aux liens affectifs tissés dans le pays étranger d'origine avec la famille élargie plutôt que par la pratique quotidienne au sein d'une communauté ethnique.

Dans les trois pays, l'identification au pays étranger d'origine est le fruit de la manière dont politiques publiques, parcours migratoires et liens sociaux tissés dans ce pays s'articulent. En cas d'absence de transmission parentale, et de migration passée dans le pays étranger d'origine, les liens symboliques tissés grâce à l'affection ressentie à l'égard de membres de la famille sont le dernier rempart avant que toute identification nationale disparaisse.

Cette dimension est d'importance dans le cas français pour les raisons suivantes. Premièrement, le modèle républicain d'intégration concevant le migrant comme un citoyen en devenir a sans doute eu pour conséquence que les familles mixtes s'enracinent en France. Chez les enfants de couples mixtes rencontrés en Allemagne et au Royaume-Uni, la transmission parentale a été moins fréquente, et ce, du fait du parcours migratoire parental plus souvent marqué par la rupture avec le pays étranger d'origine. De même, la migration au pays étranger d'origine y est moins fréquente. Les 
liens avec le pays étranger d'origine y sont donc plus ténus. L'identification à l'égard du pays étranger d'origine y est donc peu pratiquée.

De plus, le «registre d'identification nationale» politique est propre au cas français (Unterreiner, 2015b). Parallèlement au fait de se définir nationalement en référence à sa culture ou à sa socialisation, l'identification à la nation française se fait en tant que citoyen français ayant des droits et des devoirs. Le cumul de ces trois facteurs a pour conséquence que les enfants de couples mixtes placent l'identité française au premier plan, mais peuvent se dire «d'origine $\mathrm{x}$ ou y» en référence aux liens affectifs tissés dans le pays d'origine par le canal de la filiation. Ainsi, en France, c'est principalement par l'émotion, et le caractère symbolique de l'identification au pays étranger d'origine, que les identités multiples se maintiennent.

Tandis qu'en Allemagne, les liens affectifs intrafamiliaux renforcent les identités multiples transmises par les familles mixtes, dont le parent migrant était, jusque dans les années 1990 au moins, considéré comme un «invité». Les parents nés à l'étranger y ont tous migré à l'âge adulte, à une exception près, et ce, contrairement au cas français où les migrants enfants ne sont pas rares. Les projets de retour, de même que les migrations familiales pendulaires, y sont plus représentés que dans les deux autres pays, expliquant ainsi pourquoi la transmission parentale de référents identitaires renvoyant au pays étranger d'origine y est plus forte qu'ailleurs. Parallèlement, les migrations individuelles sont, tout comme au Royaume-Uni, plus fréquentes qu'en France. Ainsi, les parcours migratoires familiaux et individuels ont forgé des identités «d'héritier» ou d' «étranger», s'identifiant uniquement à un pays autre que le pays de résidence (Unterreiner, 2015a). Les enfants de couples mixtes résidant en Allemagne ont donc les moyens de pratiquer leur identification à leur pays étranger d'origine. Le lien affectif avec le pays étranger d'origine et le caractère symbolique qui en résulte renforcent donc des registres d'identification préexistants.

De plus, dans le contexte du débat sur la double nationalité, une volonté de reconnaissance officielle de l'identité d'héritier est apparue parmi les enquêtés rencontrés en Allemagne. En 1999, le CDU/CSU24 a lancé une campagne contre la double nationalité dans le Land de Hesse notamment, région où j'ai effectué mon enquête. La coalition au pouvoir a alors accepté l'Optionsmodell du FDP25 (Green, 2005: 939-940). Celui-ci a été adopté, exigeant que les individus nés en 2000 ou après en Allemagne de deux parents étrangers, qui en vertu du jus soli étaient allemands, et en vertu du jus sanguinis avaient la nationalité de leurs parents étrangers, choisissent entre ces deux nationalités entre 18 et 23 ans. Cette réforme semble avoir suscité chez les enfants de couples mixtes résidant en Allemagne un rapport différent à leur double nationalité, alors même que ceux qui ont un parent allemand ne sont pas concernés par cette réforme. Pour certains, la perspective d'un choix nécessaire fondée sur une interprétation erronée de la loi a pu conduire à un détachement de la valeur symbolique de la

24. Acronymes de l'Union chrétienne-démocrate d'Allemagne (CDU) et de l'Union chrétienne sociale représentée en Bavière (CSU).

25. Parti libéral démocrate. 
nationalité. Tandis que d'autres ont développé une stratégie défensive à l'égard de la politique mettant en cause le principe de la double nationalité et une volonté de reconnaissance de leur identité d'héritier. Ainsi, plus qu'ailleurs, les enfants de couples mixtes accordent une importance symbolique à la double nationalité. Dans le cas allemand, l'articulation entre politiques publiques, migrations familiales et individuelles, et lien familial conduit donc les enfants de couples mixtes à s'identifier à leur pays étranger d'origine de manière à la fois symbolique et pratiquée.

Enfin au Royaume-Uni, l'identification symbolique à son pays étranger d'origine apparaît comme un registre d'identification supplémentaire parmi une multitude d'autres. La définition d'un sentiment d'appartenance nationale y est plus ambiguë qu'en France et en Allemagne. À la nation britannique s'ajoutent les nations anglaise, écossaise, galloise et irlandaise. De plus, jusqu'en 1981, la nationalité britannique n'était pas fondée sur l'ascendance ou la naissance au Royaume-Uni. La loi sur la nationalité de 1948 avait en effet confirmé le statut de sujet de la Couronne dans l'ensemble de l'Empire britannique sans soumettre ce statut à un quelconque critère d'appartenance nationale. Parallèlement, contrairement à la France et à l'Allemagne, la jouissance de droits politiques n'est pas limitée aux citoyens britanniques, mais aux sujets de la Couronne résidant sur le territoire britannique. Ainsi, les résidents citoyens de l'Union européenne peuvent voter aux élections locales et européennes, tandis que ceux ayant la nationalité d'un pays du Commonwealth ont les mêmes droits politiques que les résidents au Royaume-Uni de nationalité britannique. La communauté politique ne recouvre donc pas strictement la communauté nationale au Royaume-Uni, ce qui rend les frontières de cette dernière moins lisibles. En parallèle, l'«ethnicité» a été fortement valorisée dans les discours publics. Être citoyen britannique, tout comme la binationalité, est donc perçu sous un angle pragmatique plus fréquemment que dans les cas français et allemand (Unterreiner, 2015b). Relativement au caractère ambigu de la définition de la nation, l'identité nationale y est, plus qu'ailleurs, fondée sur les convictions politiques des enfants de couples mixtes, renforçant ainsi le caractère symbolique de l'identification au pays étranger d'origine. S'identifier à son pays étranger d'origine au nom de l'affectif est une option d'identification parmi une multitude d'autres. Tandis qu'en Allemagne, l'affectif renforce l'identification d'héritier ou d'étranger, et qu'en France, il a une fonction de compensation de l'absence de registres d'identification à l'égard d'autres groupes de référence que la nation française.

\section{CONCLUSION}

L'analyse de discours identitaires a permis de mettre au jour le regard que portent les enquêtés sur leur (s) identité (s) nationale (s), et comment ils lient identités et pratiques. Si les recherches antérieures portant sur les secondes générations d'immigrés ont souligné l'importance de la réaction aux discriminations, et de l'intégration communautaire, la présente enquête a permis de mettre au jour une troisième dimension conduisant à l'identification au pays étranger d'origine: le lien de filiation. Hormis les enfants de couples mixtes qui ont résidé à l'étranger, c'est en référence à la transmis- 
sion parentale et aux liens affectifs au sein de la famille élargie que l'identification à l'égard de son pays étranger d'origine se fait.

Mais la question des pratiques effectives des enquêtés reste ouverte, car le discours est par définition le produit de leur rapport subjectif à l'égard de celles-ci. Si des recherches ethnographiques à venir permettaient de répondre à cet autre questionnement, j'ai ici mis au jour que l'identification nationale à l'égard du pays étranger d'origine passe par l'affectif et le lien de filiation, à l'exception des enquêtés qui ont eux-mêmes vécu dans ce pays et y ont toujours des liens forts.

Cette identification nationale passe rarement par la «communauté» migrante. En effet, la participation à des activités propres à la communauté migrante est rare. Et si pression extérieure il y a, celle-ci est minoritaire et provient des parents plutôt que de membres de la communauté extérieurs à la famille nucléaire. Elle est donc vécue avant la prise d'indépendance de l'enfant de couple mixte à l'égard de ses parents. Ce qui est perçu par les enfants de couples mixtes comme une contrainte correspond aux activités choisies par les parents sans leur accord, tel l'apprentissage de la langue du parent migrant lors de cours du soir avec ces derniers, ou encore la fréquentation plus ou moins régulière d'institutions communautaires étant enfants. La pression communautaire s'il en est, c'est-à-dire si le parent migrant est intégré dans la communauté migrante, s'exerce donc plus à l'égard de ce dernier que de l'enfant de couple mixte. Parler d'un repli communautaire concernant les enfants de couples mixtes serait de ce fait erroné. Leur identification en référence à leur pays étranger d'origine est fondée sur l'émotion et est choisie plutôt que contrainte.

L'identité nationale affective est le dernier rempart avant que les origines nationales étrangères ne soient reléguées au passé et ne soient plus source d'identification pour l'enfant de couple mixte. Mettre en avant une «identité d'enraciné» (Unterreiner, 2015a), c'est-à-dire au nom des liens forts tissés dans le pays de résidence, ne présuppose donc pas de l'absence d'identification «symbolique» ou affective au pays d'origine de son parent migrant. Contrairement aux conclusions des travaux issus de la Première École de Chicago, la présente recherche a mis en avant qu'identification au pays de résidence et identification au pays d'origine ne sont pas incompatibles, hiérarchisées ni mises sous tension. Mais elles répondent à des logiques discursives différentes. C'est l'articulation des liens sociaux tissés dans les pays d'origine et de résidence et l'importance accordée à ces liens par l'individu qui permettent d'expliquer la manière dont l'enfant de couple mixte s'identifie nationalement.

\section{RÉSUMÉ}

Étudier l'identité des enfants de couples mixtes permet d'éclairer les débats contemporains portant sur le contrôle de l'immigration et de l'intégration des populations issues de l'immigration. Une telle entreprise rend de plus visibles les facteurs susceptibles d'influencer l'identité nationale. Cet article s'attache à analyser le caractère «pratiqué» ou «symbolique» de l'identification nationale en référence au pays d'origine du parent migrant, à partir des discours identitaires d'une centaine d'enfants de couples mixtes rencontrés en France, en Allemagne et au Royaume-Uni. L'identification des enfants de couples mixtes à leurs origines 
nationales étrangères semble symbolique, c'est-à-dire élective plutôt que due à la pression communautaire. Elle se fonde sur l'affectif et le lien de filiation avant tout. Si au Royaume-Uni, cette identité symbolique s'ajoute à une multitude d'autres identités, en Allemagne, elle enrichit le sentiment d'appartenance au pays étranger d'origine existant. En France, elle compense l'absence d'identifications autres qu'en référence à la nation française.

Mots clés: enfants de couples mixtes, identité nationale, identité symbolique, communauté, lien de filiation

\section{ABSTRACT}

Studying the identity of children "nationally" mixed couples helps shed light on contemporary debates on the control of immigration and integration of immigrant populations. Such an endeavor helps clarify the factors that can shape national identity. This article analyzes the "practical'or "symbolic" character of national identity with respect to the country of origin of the migrant parent, based on the identity discourse of about one hundred children mixed couples interviewed in France, Germany and the United Kingdom. The identification that children mixed couples have with their foreign country of origin seems to be symbolic, i.e., elective rather than because of community pressure. It is based above all on emotional connections and the childparent relationship. While in the United Kingdom this symbolic identity is aggregated with numerous other identities, in Germany it enriches the feeling of belonging to the existing foreign country of origin. In France, it compensates for the absence of identifications other than those that refer to French nationhood.

Key words: children "nationally" mixed couples, national identity, symbolic identity, community, parent-child ties

\section{RESUMEN}

Estudiar la identidad de los hijos de parejas mixtas permite aclarar los debates contemporáneos acerca del control de la inmigración y de la integración de la población inmigrante. Tal empresa hace más visible los factores susceptibles de influir en la identidad nacional. Este artículo busca analizar el carácter "práctico" o "simbólico" de la identificación nacional con referencia al país de origen del padre o de la madre inmigrante, a partir de los discursos identitarios de un centenar de hijos de parejas mixtas entrevistados en Francia, Alemania y el Reino Unido. La identidad de los hijos de parejas mixtas y sus orígenes nacionales y extranjeros parece simbólica, es decir, electiva antes que debida a la presión comunitaria. Ésta se funda ante todo en lo afectivo y en el vínculo de filiación. Si en el Reino Unido esta identidad simbólica se suma a una multitud de otras identidades, en Alemania enriquece el sentimiento de pertenencia al país extranjero de origen y en Francia compensa la ausencia de identificaciones diferentes a la de la nación francesa.

Palabras clave: hijos de parejas mixtas; identidad nacional; identidad simbólica; comunidad; vínculo de filiación 


\section{BIBLIOGRAPHIE}

AlbA, R. (1990), Ethnic Identity: The Transformation of White America, New Haven et Londres, Yale University Press.

Ali, S. (2003), Mixed-Race, Post-Race: Gender, Ethnicities and Cultural Practices, Oxford, Berg.

Anderson, B. (2006 [1991]), Imagined Communities: Reflections on the Origin and Spread of Nationalism, New York, Verso.

Aspinall, P. et M. Song (2013), Mixed Race Identities, Londres, Palgrave and Macmillan.

BARTH, F. (1969), «Introduction», in Barth, F. (dir.), Ethnic Groups and Boundaries: The Social Organization of Culture Difference, Londres, George Allen \& Unwin, p. 9-38.

Benson, S. (1981), Ambiguous Ethnicity: Interracial Families in London, Cambridge, Cambridge University Press.

Caballero, C., R. Edwards et S. Puthussery (2008), Parenting «Mixed» children: Negotiating Difference and Belonging in Mixed Race, Ethnicity and Faith Families, York, Joseph Rowntree Foundation.

Collet, B. (2003), «Modes d'intégration nationale et mariage mixte en France et en Allemagne: à propos d'un processus de construction de comparaison internationale», in LALLEMENT, M. et J. SPURK (dir.), Stratégies de la comparaison internationale, Paris, CNRS Éditions, p. 233-247.

European Forum for Migration Studies (2001), Effectiveness of National Integration Strategies Towards Second Generation Migrant Youth in a Comparative European Perspective - EFFNATIS. Rapport final à la Commission Européenne. Bamberg, Université de Bamberg.

Gans, H. (1979), «Symbolic Ethnicity: The Future of Ethnic Groups and Cultures in America», Ethnic and Racial Studies, vol. 2, n 1, p. 1-20.

Gordon, M. (1964), Assimilation in American Life: The Role of Race, Religion, and National Origins, New York, Oxford University Press.

Green, S. (2005), «Between Ideology and Pragmatism: The Politics of Dual Nationality in Germany», International Migration Review, vol. 39, n 4, p. 921-952.

Khounani, P. (2000), Binationale Familien in Deutschland und die Erziehung der Kinder: Eine Vergleichuntersuchung, Frankfurt am Main, Peter Lang.

LAhire, B. (1998), L'homme pluriel: Les ressorts de l'action, Paris, Nathan.

Layton-Henry, Z. (1985) «Great Britain», in Hammar, T. (dir.), European Immigration Policy: A Comparative Study, Cambridge, Cambridge University Press, p. 89-126.

Le Gall, J. (2003), Transmission identitaire et mariages mixtes: Recension des écrits, Montréal, Groupe de recherche ethnicité et société du Centre d'études ethniques.

Le Gall, J. et D. Meintel (2011), «Liens transnationaux et transmission intergénérationnelle: le cas des familles mixtes au Québec», Autrepart, n 57-58, p. 127-143.

Lesbet, D. et G. VArro (1995), «Discours d'adolescents», in VArro, G. (dir.), Les couples mixtes et leurs enfants en France et en Allemagne, Paris, Armand Colin, p. 155-181.

Meintel, D. (1992), «L'identité ethnique chez les jeunes Montréalais d'origine immigrée». Sociologie et sociétés, vol. $24, \mathrm{n}^{\circ} 2$, p. 73-89.

(2002), «Transmitting Pluralism: Mixed Unions in Montreal», Canadian Ethnic Studies, vol. 34, $\mathrm{n}^{\circ} 3$, p. 99-120.

Merton, R. K. (1997 [1957]), Éléments de théorie et de méthode sociologique, Paris, Armand Colin.

Mucchielli, A. (2009 [1986]), L’identité, Paris, PUF.

Paugam, S. (2008), Le lien social, Paris, PUF.

Pollock, D. C. et R. E. Van Reken (2009 [1999]), Third Culture Kids: Growing Up Among Worlds, Boston, Nicholas Brealey Publishing.

Portes, A. et R. Rumbaut (2001), Legacies: The Story of the Immigrant Second Generation. Berkeley et New York, University of California Press et Russell Sage Foundation.

Puzenat, A. (2008), «Le vécu de la mixité conjugale chez les couples franco-maghrébins et la transmission identitaire aux enfants", Diversité urbaine, vol. 8, n 1, p. 113-128. 
Ramos, E. (2006), L'invention des origines: Sociologie de l'ancrage Identitaire, Paris, Armand Colin.

Saenz, R., Hwang, S.-S., Aguirre, B. E. et R. N. Anderson (1995), «Persistence and Change in Asian Identity among Children of Intermarried Couples», Sociological Perspectives, vol. 38, n 2, p. 175-194. SAFI, M. (2007), «Le devenir des immigrés en France: Barrières et inégalités», Paris, EHESS.

Schnapper, D. (1991), La France de l'intégration: Sociologie de la nation en 1990, Paris, Éditions Gallimard. (1992), L'Europe des immigrés, Paris, François Bourin.

Somerville, K. (2008), "Transnational Belonging among Second Generation Youth : Identity in a Globalized World», Journal of Social Sciences, $n^{\circ} 10$, p. 23-33.

Song, M. (2003), Choosing Ethnic Identity, Cambridge, Polity Press.

- (2010), «What Happened after Segmented Assimilation? An Exploration of Intermarriage and

"Mixed Race”Young People in Britain", Ethnic and Racial Studies, vol. 33, n 7, p. 1194-1213.

Stonequist, E. (1965 [1937]), The Marginal Man: A study in Personality and Culture Conflict, New York, Russell and Russell Inc.

Tizard, B. et A. Phoenix (1993), Black, White or Mixed Race, Londres, Routledge.

Unterreiner, A. (2012), "Liens sociaux et construction identitaire des enfants de couples mixtes: une étude comparée en France, en Allemagne et au Royaume-Uni», Doctorat, EHESS.

Unterreiner, A. (2015a), «Enfants de couples mixtes: Liens sociaux et identités», Rennes, Presses Universitaires de Rennes.

- (2015b), From Registers to Repertoires of Identification in National Identity Discourses : «A Comparative Study of Nationally Mixed People in France, Germany and the United Kingdom», Studies in Ethnicity and Nationalism, vol. 15, $\mathrm{n}^{\circ} 2$, p. 251-271.

- (2014), «La transmission de la langue du parent migrant au sein des familles mixtes: une réalité complexe perçue à travers le discours de leurs enfants ", langage \& société, nº 147, p. 97-109.

Waters, M. (1990), Ethnic Options: Choosing Identities in America, Berkeley et Los Angeles, University of California Press.

(1994), «Ethnic and Racial Identities of Second-Generation Black Immigrants in New York City», International Migration Review, vol. 28, $\mathrm{n}^{\circ}$ 4, p. 795-820.

Wolf, D. (2002), «There's No Place Like "Home": Emotional Transnationalism and the Struggles of Second-Generation Filipinos", in P. Lewitt et M. Waters (dir.), The Changing Face of Home: The Transnational Lives of the Second Generation, New York, Russell Sage Foundation, p. 255-294. 